\title{
Hierarchically aligned fibrin nanofiber hydrogel accelerated axonal regrowth and locomotor function recovery in rat spinal cord injury
}

This article was published in the following Dove Press journal: International Journal of Nanomedicine

\author{
Shenglian Yao ${ }^{1,2}$ \\ Shukui $\mathrm{Yu}^{3}$ \\ Zheng $\mathrm{CaO}^{2}$ \\ Yongdong Yang ${ }^{2,4}$ \\ Xing $\mathrm{Yu}^{4}$ \\ Hai-Quan $\mathrm{Mao}^{5}$ \\ Lu-Ning Wang' \\ Xiaodan Sun ${ }^{2}$ \\ Lingyun Zhao ${ }^{2}$ \\ Xiumei Wang ${ }^{2}$ \\ 'School of Materials Science and \\ Engineering, University of Science and \\ Technology Beijing, Beijing, China; ${ }^{2}$ Key \\ Laboratory of Advanced Materials \\ of Ministry of Education, School of \\ Materials Science and Engineering, \\ Tsinghua University, Beijing, China; \\ ${ }^{3}$ Department of Otolaryngology-Head \\ and Neck Surgery, Beijing Friendship \\ Hospital, Capital Medical University, \\ Beijing, China; ${ }^{4}$ Department of \\ Orthopedics, Dongzhimen Hospital, \\ Beijing University of Chinese \\ Medicine, Beijing, China; ${ }^{5}$ Department \\ of Materials Science and Engineering, \\ Johns Hopkins University, Baltimore, \\ MD, USA
}

Correspondence: Xiumei Wang Key Laboratory of Advanced Materials of Ministry of Education, School of Materials Science and Engineering, Tsinghua University, YiFu Technology and Science Building 2804, Beijing, 100084, China

Tel +86 I0 62782966

Fax +86 I0 6277 II60

Emailwxm@mail.tsinghua.edu.cn
Background: Designing novel biomaterials that incorporate or mimic the functions of extracellular matrix to deliver precise regulatory signals for tissue regeneration is the focus of current intensive research efforts in tissue engineering and regenerative medicine.

Methods and results: To mimic the natural environment of the spinal cord tissue, a threedimensional hierarchically aligned fibrin hydrogel (AFG) with oriented topography and soft stiffness has been fabricated by electrospinning and a concurrent molecular self-assembling process. In this study, the AFG was implanted into a rat dorsal hemisected spinal cord injury model to bridge the lesion site. Host cells invaded promptly along the aligned fibrin hydrogels to form aligned tissue cables in the first week, and then were followed by axonal regrowth. At 4 weeks after the surgery, neurofilament (NF)-positive staining fibers were detected near the rostral end as well as the middle site of defect, which aligned along the tissue cables. Abundant NF- and GAP-43-positive staining indicated new axon regrowth in the oriented tissue cables, which penetrated throughout the lesion site in 8 weeks. Additionally, the abundant blood vessels marked with RECA-1 had reconstructed within the lesion site at 4 weeks after surgery. BassoBeattie-Bresnahan scoring showed that the locomotor performance of the AFG group recovered much faster than that of blank control group or the random fibrin hydrogel (RFG) group from 2 weeks after surgery. Furthermore, diffusion tensor imaging tractography of MRI confirmed the optimal axon fiber reconstruction compared with the RFG and control groups.

Conclusion: Taken together, our results suggested that the AFG scaffold provided an inductive matrix for accelerating directional host cell invasion, vascular system reconstruction, and axonal regrowth, which could promote and support extensive aligned axonal regrowth and locomotor function recovery.

Keywords: spinal cord injury, nerve regrowth, fibrin hydrogel, aligned structure, soft stiffness

\section{Introduction}

Spinal cord injury (SCI) is extremely difficult to repair and often leads to permanent neurological impairments. Unlike peripheral nervous system (PNS) injury, the spontaneous axonal regeneration after SCI is very limited, and unfortunately there are no effective therapeutic methods capable of completely restoring neurological function so far. The failure of spinal cord regeneration can be attributed to the extremely limited regenerative capacity of neurons and an inhibitory hostile microenvironment caused by pathological sequelae of the initial trauma, which introduces a physical and chemical barrier to regeneration, such as glial scar formation, neurotrophic factor deficiency, cystic cavity, and myelin-associated inhibitors. ${ }^{1-3}$ Therefore, one promising therapeutic strategy for the treatment of SCI is based on the principle of tissue engineering and 
regenerative medicine to engineer novel biomaterials for breaking the physical and chemical barrier to regeneration and guiding axonal regeneration..$^{47}$ Here, biomaterial scaffold serves as a beneficially regenerative microenvironment that could deliver regulatory signals to instruct cell fate and related regenerative processes.

It has been clearly proved that biomaterial-based control of the cell may occur through multiple physical and chemical cues, such as biomaterial's mechanical properties, topography, surface chemistry, or biological ligands. For example, early evidence showed that the stiffness or elasticity of biomaterial can have a major influence on cell behaviors such as migration, proliferation, and differentiation..$^{8-10}$ To mimic the stiffness of native nerve extracellular matrix (ECM), a variety of hydrogels with mechanical properties that can be tuned to be similar to those of native nerve tissue have been investigated as ideal candidates for spinal cord regeneration, showing positive roles in inducing stem cell neurogenic differentiation in vitro and axonal regrowth in vivo with significant and long-lasting motor functional recovery. ${ }^{11-19}$

Unfortunately, most of these hydrogels are isotropic in architecture and lack oriented topography. Spinal cord tissue, especially the white matter that is composed of well-aligned axon tracts, has hierarchically oriented tissue structures ranging from a single neural axon to nerve fibers. ${ }^{20}$ Therefore, the aligned architecture also works as an important topographical guidance cue to bridge the lesion site and encourage axon regeneration. ${ }^{21}$ In order to impose aligned guidance cue on hydrogels, all kinds of aligned microstructures/ macrostructures have been created in hydrogel scaffolds using the methods like porogen leaching, freeze drying, crystal templating, multiphoton lithography, and molding. ${ }^{1,22,23}$ For example, Gros et al fabricated an agarose hydrogel scaffold with strictly linear guidance channels, which could support linear growth of long-tract axons of the injured spinal cord and promote axonal egress from lesion sites. ${ }^{24}$ The anisotropic alginate-based hydrogels with continuous linear channels were able to integrate into a spinal cord lesion and provide a stable physical passageway to promote axonal regeneration in a highly oriented fashion. ${ }^{13,25,26} \mathrm{In}$ comparison to the linear channel structure, the aligned fiber structure was shown to be more similar to the nerve tissue, and designed for nerve regeneration. ${ }^{27,28}$ For instance, linearordered collagen fibers with the addition of brain-derived neurotrophic factor provided effective bridge and stimulation for neural regeneration, with recovery of locomotor function in both rat and canine SCI models. ${ }^{29-31}$ Similarly, the aligned fibrous structure in nanoscale also was introduced to the nerve regeneration. Nguyen et al incorporated aligned poly(caprolactone-co-ethyl ethylene phosphate) nanofibers within a collagen hydrogel in a three-dimensional (3D) configuration, which provided topographical signals for effectively directing neurite extensions and supporting remyelination within the lesion sites. ${ }^{32}$ Berns et al prepared an aligned self-assembled peptide amphiphile hydrogel with a nanoscale fibrous structure, and the results demonstrated that the scaffold could guide the growth of oriented neurites. ${ }^{33}$ Therefore, the fabrication of aligned nanofiber structure in hydrogel is thought to be useful in nerve tissue engineering by delivering joint regulatory signals of low elasticity and oriented topography simultaneously.

In our previous work, a 3D aligned fibrin hydrogel (AFG) with oriented structure and low elasticity $(\sim 1.5 \mathrm{kPa})$ was developed to direct mesenchymal stem cell neurogenic differentiation and rapid neurite outgrowth. ${ }^{34}$ Fibrin is one of the most important proteins involved in normal hemostasis, wound healing, and tissue regeneration. ${ }^{35-38}$ Especially during the initial regenerative process of PNS, acellular aligned fibrin cable formation occurs spontaneously within the hollow nerve guidance conduit, bridging the proximal and distal nerve stumps. Following this process, Schwann cells, endothelial cells, and fibroblasts migrate, align, and proliferate longitudinally along the align fibrin cable to form a biological tissue cable to guide axonal sprouting. ${ }^{39}$ Furthermore, the fibrin hydrogel also showed potential for promoting axonal regrowth in SCI regeneration..$^{40}$ Therefore, fibrin hydrogels have been widely used in nerve regeneration. We herein applied the fibrin nanofiber hydrogel in imparting topographical and mechanical signals to enhance nerve regeneration after SCI.

In this study, we implanted the AFG into a rat dorsal hemisected SCI to bridge the lesion site and induce neural tissue regrowth. The cellular and axonal regrowth were evaluated by immunohistochemistry from 1 week after the surgery, and the regenerating axons in the lesion site were further observed by diffusion tensor imaging (DTI) and transmission electron microscopy (TEM). Hindlimb locomotor function of the rat was evaluated by the Basso-Beattie-Bresnahan (BBB) scale.

\section{Materials and methods Preparation and characterization of fibrin hydrogels}

Fibrinogen, thrombin, poly(ethylene oxide) (average molecular weight ca 4,000 kDa), and alginate were purchased from Sigma-Aldrich, St. Louis, MO, USA. The AFG was fabricated through electrospinning according to previous work. ${ }^{34,41}$ 
The electrospun AFG was gathered to form a $2 \mathrm{~mm}$ thick halfcylindrical bundle. Then, the surface of AFG was coated with alginate by dipping into $1 \%$ alginate solution and crosslinking with $50 \mathrm{mMCaCl}_{2}$ solution. The random fibrin hydrogel (RFG) was fabricated by the direct reaction of $1 \%$ fibrinogen and 20 units/mL of thrombin, and then also coated with alginate.

To observe the microstructure of the fibrin scaffolds, the samples were fixed with $2.5 \%$ glutaraldehyde for $2 \mathrm{~h}$ at $4^{\circ} \mathrm{C}$, then dehydrated through graded concentrations of ethanol $(30,50,60,70,80$, 90,95 , and $100 \%$ ), and finally critical-point dried with $\mathrm{CO}_{2}$. The fresh surfaces of the samples were imaged with a field-emission scanning electron microscope (SEM; JEOL 6700F; JEOL, Tokyo, Japan) after being sputter coated with a layer of $\mathrm{Au}$.

\section{Rat T9 dorsal hemisected $\mathrm{SCl}$}

Animal experiments were performed according to the Guide for the Care and Use of Laboratory Animals of the National Institutes of Health and were approved by the Animal Care and Use Committee at Capital Medical University (Beijing, China). Adult female SD rats (200-230 g) were anesthetized by intraperitoneal injection with $6 \%$ chloral hydrate at $6 \mathrm{~mL} / \mathrm{kg}$. After routine shaving and cleaning with povidone iodine, surgery was performed under sterile conditions. After dorsal laminectomy at the T9-T10 vertebral level, a portion of the spinal cord ca. $4 \mathrm{~mm}$ long and $1.5 \mathrm{~mm}$ in dorsal thickness was removed to create a hemisected lesion (Figure 1). After hemostasis, a fibrin hydrogel scaffold (AFG, RFG) was implanted into the lesion site to bridge the defect; $0.9 \%$ saline was used as a blank control. For the AFG group, the aligned fibrin fibers were positioned parallel to the long axis of the spinal cord. After the operation, $1 \mathrm{~mL}$ of penicillin was injected subcutaneously to prevent infection. Bladders were emptied manually by pressing twice a day until bladder function recovered.

\section{Tissue preparation and immunohistochemistry}

After 1, 2, 4, and 8 weeks following implantation, the rats ( $n=4$ at each time point for each group) were anaesthetized and then perfused with $100 \mathrm{~mL}$ of $0.9 \%$ saline and $300 \mathrm{~mL}$ of cold $4 \%$ formaldehyde through the heart to fix the tissues. The spinal cord tissue (T8-T11) containing the injury site was harvested and used in histological analysis.

The tissue section containing the injury site was cryopreserved in the optimal cutting temperature compound and sliced longitudinally into $20 \mu \mathrm{m}$ thick sections using a cryostat (CM1900; Leica Microsystems, Wetzlar, Germany). For immunofluorescence staining, the sections were incubated for $1 \mathrm{~h}$ at room temperature in PBS with 10\% goat serum and
$0.3 \%$ Triton $\mathrm{X}-100$ to block nonspecific binding and improve the penetration of the antibody. Next, the tissue sections were incubated with primary antibodies at $4^{\circ} \mathrm{C}$ overnight. Then, after washing three times with PBS, they were incubated with secondary antibodies for $1 \mathrm{~h}$ at room temperature in the dark. Finally, the tissue section was mounted using a drop of mounting medium containing 4',6-diamidino-2-phenylindole (DAPI; Abcam, Cambridge, UK). The following immunocytochemical markers were used: rabbit anti- $\beta$-tubulin III $(1: 1,000)$ to label the endogenous neuron; mouse anti-neurofilament (NF) $(1: 1,000)$ and rabbit anti-GAP43 (1:500) antibodies to detect axon regrowth; and mouse anti-endothelial cell antibody (RECA-1) (1:500) to investigate vascularization in the lesion site. The anti-rabbit (1:800, 594; Thermo Fisher Scientific, Waltham, MA, USA) and anti-mouse (1:800, 488; Thermo Fisher Scientific) secondary antibodies were used correspondingly.

F-actin staining was employed to investigate the migration of endogenous cells into the hydrogel in the first week. For F-actin staining, the sections were incubated in PBS with $1 \%$ bovine serum albumin and $0.1 \%$ Triton $\mathrm{X}-100$ for $1 \mathrm{~h}$ at room temperature to block nonspecific binding and improve the penetration of the antibody, and then stained with F-actin marker rhodamine-phalloidin (Dojin, Kurume, Japan) at room temperature for $40 \mathrm{~min}$, washed three times with PBS, and then mounted with a drop of mounting medium containing DAPI.

The slides were examined by laser confocal fluorescence microscopy (LSM 780; Carl Zeiss Meditec AG, Jena, Germany) using the tile scan function.

\section{DTI acquisition and analysis}

DTI datasets were obtained at 16 weeks after injury. The animals ( $\mathrm{n}=4$ for each group) were anesthetized with intravenous pentobarbital sodium at $30 \mathrm{mg} / \mathrm{kg}$. Magnetic resonance imaging (MRI) data on the rat SCI were acquired using a 7.0 T Bruker Bio Clin Spin Animal MRI System (Bruker, Billerica, MA, USA) with a specialized coil for scanning rats. The DTI experiments were performed using the single-shot spin-echo echo-planar imaging sequence. The imaging parameters were as follows: field of view $=36 \times 30 \mathrm{~mm}$, acquisition matrix $=128 \times 128$, slice thickness $=0.8 \mathrm{~mm}$, slice number $=25$, inter-slice gap $/$ slice thickness $=30 \%$, echo time $=5,000 \mathrm{~ms}$, repetition time $=60 \mathrm{~ms}$, corresponding to various non-collinear and non-coplanar diffusion directions with $b$-value $=600 \mathrm{~s} / \mathrm{mm}^{2}$.

Software Diffusion Toolkit version 0.6 (Wang R, Wedeen VJ, TrackVis.org, Martinos Center for Biomedical Imaging, Massachusetts General Hospital, Boston, MA, USA) was used for the tensor reconstruction and fiber tractography (http://www.trackvis.org). Fractional anisotropy (FA) maps, 
A

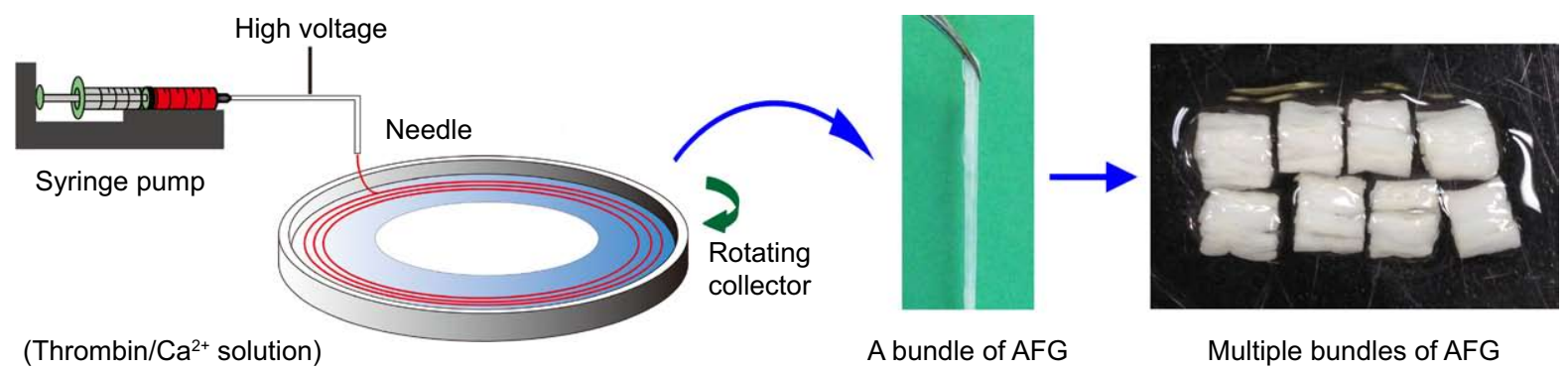

B
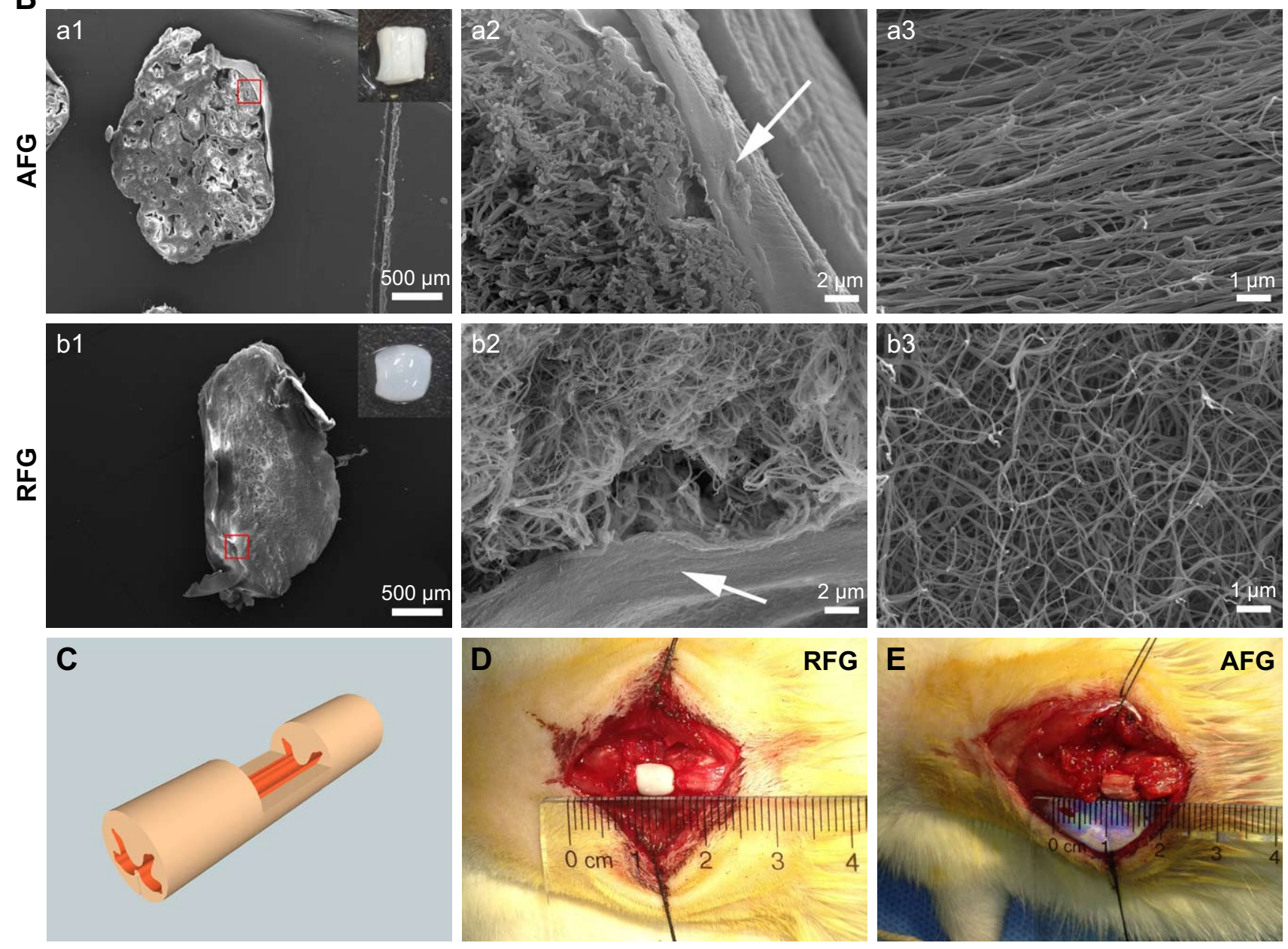

Figure I Fibrin hydrogel scaffold and rat T9 dorsal hemisected SCI model. (A) The AFG fabrication process. (B) (al-a2) and (bI-b2) SEM images of the cross sections of AFG and RFG scaffold reveal the hierarchically aligned structure of AFG and random fibrous structure of RFG, respectively; the insert pictures in (al) and (bI) display gross images of the implanted AFG and RFG scaffolds; the arrows in (a2) and (b2) show the alginate film in the surface of the hydrogel for adhesive prevention. (a3 and b3) SEM images of the longitudinal sections of AFG and RFG scaffolds, demonstrating the aligned and random nanoscale fibrous structure, respectively. (C) Schematic illustration of the rat T9 dorsal hemisected model, and RFG and AFG scaffolds implantation (D and E).

Abbreviations: AFG, aligned fibrin hydrogel; RFG, random fibrin hydrogel; SCI, spinal cord injury; SEM, scanning electron microscope.

three eigenvalues $(\lambda 1, \lambda 2, \lambda 3)$, and their corresponding eigenvectors were acquired. Tract visualization and DTI metrics quantification were performed using Track Vis ver. 0.5 (Harvard Medical School, Boston, MA, USA). The focal point of the SCI was established on the FA map at T9. A 2 mm long segment was then measured in both rostral and caudal directions from the epicenter and further subdivided at $0.4 \mathrm{~mm}$ increments. After the regions of interest were delineated, FA values were generated from the corresponding FA maps by outlining the entire cord parenchyma in the axial plane; this process resulted in a numerical measure and FA values averaged within groups. These were plotted as a function of their locations along the spinal cord.

\section{TEM observations}

After 16 weeks, the rats ( $\mathrm{n}=4$ for each group) were sacrificed for ultrastructural observation and analysis. The animals were 
perfused with $0.01 \mathrm{mM}$ PBS ( $\mathrm{pH} 7.4,37^{\circ} \mathrm{C}$ ), followed by $4 \%$ (weight/volume) paraformaldehyde and 2\% glutaraldehyde in $0.1 \mathrm{M}$ phosphate buffer ( $\mathrm{pH} 7.4,4^{\circ} \mathrm{C}$ ). Samples from the epicenter of the injured tissue were diced for further fixation in $2.5 \%$ glutaraldehyde at $4^{\circ} \mathrm{C}$ overnight, and then with $1 \%$ osmic acid for $1.5 \mathrm{~h}$. The samples were dehydrated through graded ethanol and embedded in Epoxy Embedding Medium (Sigma Aldrich) overnight, followed by polymerization at $60^{\circ} \mathrm{C}$ for $10 \mathrm{~h}$. Ultrathin sections were cut with an ultramicrotome (EM UC6; Leica Microsystems) and stained with uranyl acetate and lead citrate. Observations and photomicrographs were obtained with a TEM (Hitachi H-7650B; Hitachi, Tokyo, Japan) operated at $80 \mathrm{kV}$.

\section{Evaluation of hindlimb locomotor function}

The locomotor functions of the control, RFG, and AFG groups were evaluated weekly by open-field testing using the BBB scale. Rats were placed on a flat, $1 \mathrm{~m}$ diameter surface and observed for $4 \mathrm{~min}$. Hindlimb locomotor recovery in animals was scored by two blinded observers on the scale of 0 (no hindlimb movement) to 21 (normal mobility).

\section{Statistical analysis}

A BBB score was expressed as means \pm standard errors of means. Other results were presented as mean \pm standard deviation. Differences in histological data, including vessel counts, axon counts, axon length, and myelin thickness, were assessed for significance using Student's $t$-test. Differences in BBB scores and FA values were assessed using two-way analysis of variance with repeated measures, followed by the post hoc Bonferroni test. The significance level for all tests was set at $p$-values $<0.05$.

\section{Results}

\section{Scaffold fabrication and implantation}

A dorsal portion of the spinal cord ca. $4 \mathrm{~mm}$ long and $1.5 \mathrm{~mm}$ thick was surgically removed to create a hemisected lesion (Figure 1). The electrospun AFG fibers were stacked into a $2 \mathrm{~mm}$ wide bundle (as shown in Figure 1) and then cut into 4 mm lengths to fit the SCI injury gap. It is noted that the gross image of the assembled AFG scaffold (insert of Figure 1) was similar to the spinal cord. The elasticities of AFG and RFG were close to the ECM of nerve tissue, which were $1.57 \pm$ $0.11 \mathrm{kPa}$ and $0.36 \pm 0.02 \mathrm{kPa}$, respectively, as reported in our previous work. ${ }^{34}$ To prevent the fibrous tissue from infiltrating into the fibrin hydrogel from the dorsal side, alginate hydrogel that is not beneficial for cell adhesion was coated only on the dorsal surface of fibrin bundle. The SEM images of the transverse and longitudinal sections of the RFG scaffold (Figure 1) showed an isotropic fibrous structure. By contrast, the SEM images of the transverse and longitudinal sections of the AFG scaffold (Figure 1) showed a hierarchically aligned fibrous structure, suggesting that it could be a bridge connecting the hemisected lesion site. The rat models with AFG and RFG implantations are shown in Figure 1.

\section{Host tissue integration and rapid vascularization}

At 2 weeks, a great many of host cells infiltrated into the lesion site in the AFG and RFG groups, as indicated by the DAPI staining of cell nuclei, whereas there was no obvious tissue formation in the control group, leaving an obviously large empty in the lesion site (Figure 2A-C). The result implied that the implanted fibrin hydrogels could promote cell invasion and have good integration with surrounding host tissues. Nevertheless, there were still many cavities formed inside the regrowth tissues in the RFG group, which may be caused by the fibrin degradation and insufficient new tissue formation. The new regrowth tissue in AFG group was much denser than that in RFG group, indicating the aligned guidance could probably accelerate cell invasion and tissue regrowth. What is more, the nerve tissue under the defect in the control group underwent severe degeneration resulting in many cavities formed in the originally healthy tissue.

Rapid vascularization within the transplanted scaffold is thought to be essential in determining the success of new tissue regrowth for transporting adequate oxygen and nutrients. RECA-1 is a specific marker for endothelial cells, which form the linings of blood vessels. As shown in Figure 2D, the density of RECA-1 positive vessels of AFG group was significantly higher than that of the RFG group at 2 and 4 weeks of implantation. The details at 4 weeks are shown in Figure 2E-J. Abundant blood vessels which were labeled with RECA-1 could be clearly detected in both the RFG and AFG groups. High magnification of confocal microscopy images (Figure $2 \mathrm{~F}$ and $\mathrm{G}$ and $\mathrm{I}$ and $\mathrm{J}$ ) revealed a higher density of blood vessels and newly formed tissues in the AFG group.

\section{Aligned cell cables supported rapid axon sprouting and regrowth}

Figure 3 shows the typical immunofluorescence photographs of the regrowth tissues in the middle and rostral sites of the lesions at 1,2, and 4 weeks after implantation. 

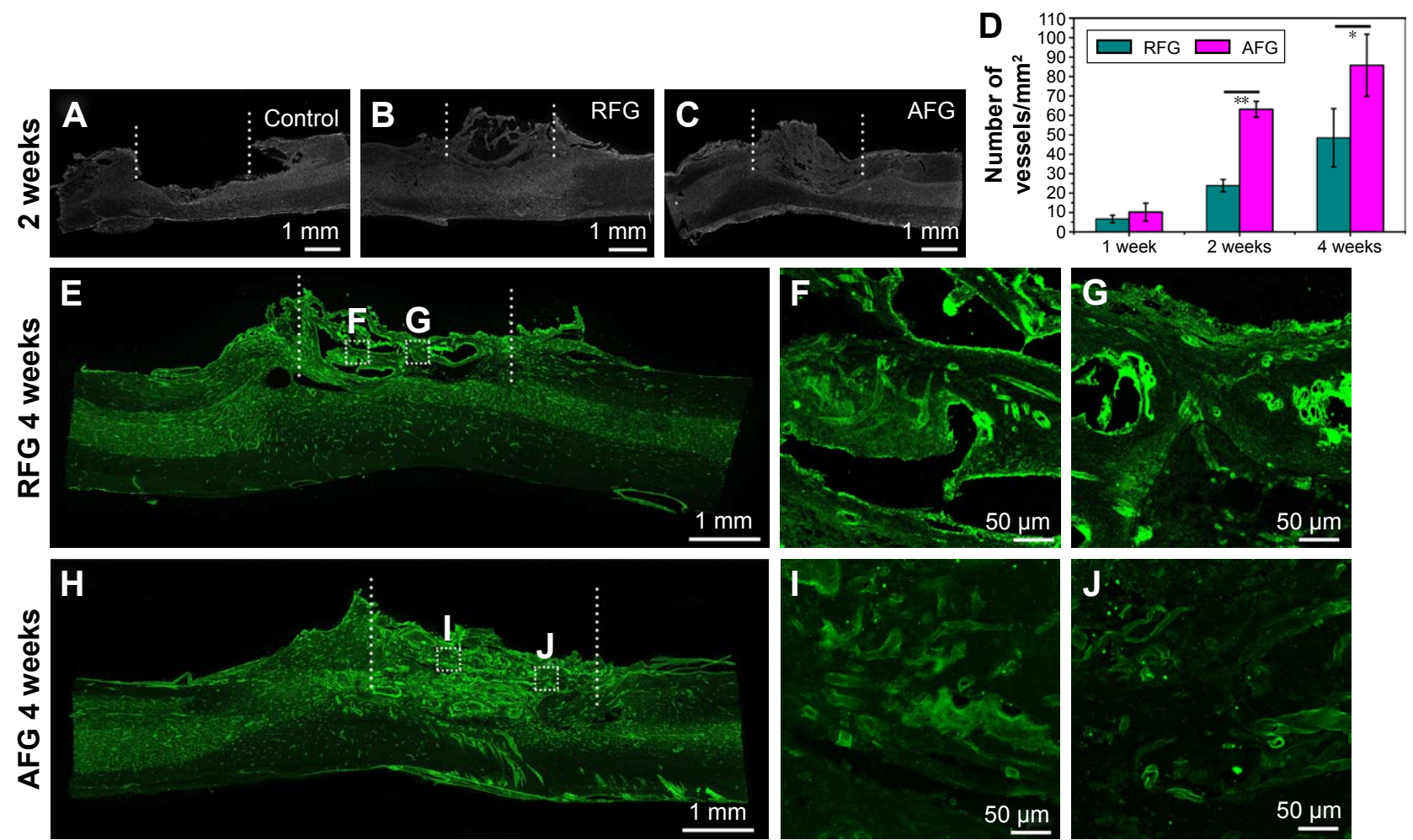

Figure 2 Vascularization in the dorsal hemisected site. (A-C) Representative sagittal sections of control, RFG, and AFG groups stained by DAPI show the tissue regeneration at 2 weeks post-surgery, and the lesion site region is labeled by dashed line. (D) Quantification of RECA-I positive vessels at the middle site of injury at I, 2, and 4 weeks after spinal cord injury. ${ }^{*} p<0.05$, ${ }^{* *} p<0.01$ for the comparison of RFG and AFG. Four individual rats per group were used for statistical analysis ( $\mathrm{n}=4$ ), and five confocal images per rats were used for the vessel counts. Immunofluorescence staining images of the T8-TI0 sagittal sections in RFG (E-G) and AFG (H-J) groups labeled by RECA-I (green).

Abbreviations: AFG, aligned fibrin hydrogel; DAPI, 4',6-diamidino-2-phenylindole; RFG, random fibrin hydrogel.

In Figure 3(a1-a3), the regrowth tissue in the injury site was labeled red by staining the F-actin and the undegenerating scaffold was black. In the RFG group, the endogenous cells randomly invaded into the implanted RFG scaffold (Figure 3A[a1]), and in the AFG group, the cells followed the fiber direction from the rostral stump toward the caudal stump of the lesion site (Figure 3A[a2 and a3]), forming strips of cell cables. At 2 weeks after implantation, with the increase of the cell invasion, the fibrin scaffolds were degraded and replaced gradually by the invading cells. As shown in Figure 3B(b1-b3), a large amount of neurons labeled with $\beta$-tubulin III were observed to form densely packed aligned cell cables (follow dashed line in Figure 3B[b2 and b3]) in the $\mathrm{AFG}$ group, while the regrowth tissue in the $\mathrm{RFG}$ group was still distributed randomly (Figure 3B[b1]). At 4 weeks post-implantation, random and short NF-positive axons were observed in the middle region of the lesion site in the RFG group. By contrast, NF-positive staining of axons longer than $30 \mu \mathrm{m}$ could be found in both the middle and the rostral regions of the lesion site in AFG group, which aligned along the cell cables. Furthermore, it is noted that the fibrin scaffolds underwent gradual biodegradation and could be proteolytically removed by regenerating tissue. ${ }^{35,36}$ The black area in Figure 3A(a1-a3) indicates the fibrin matrix that was not yet fully degraded in 1 week. With the cell invasion, fibrin was degraded by the secreted protease and replaced by the invading cells, forming aligned cell cables or random tissue in the lesion site.

The aligned cell cable formed along the hierarchically oriented AFG fibers scaffold was able to induce axonal spouting directionally and accelerate axon regrowth. Figure 4 shows the typical immunofluorescence staining of new axon regrowth at 8 weeks after the implantation. NF staining was used to label the NF, and the GAP-43 protein was associated with nerve growth as a major component of the motile "growth cones" that were formed at the tips of the elongating axons. In the control group, the defect still existed without tissue regeneration. The axons next to the caudal site had obvious degeneration, leaving many cavities or random scar tissue (Figure 4A[a1-a3]). With the fibrin scaffold implantation, the surrounding neural tissue showed little further degeneration. Notably, the host-graft interface was not obvious. For the excellent integration of fibrin scaffold and host tissue, the host cells regrew into the fibrin scaffold and replaced the scaffold structure. In addition, the regrowth axons in AFG group showed remarkable 

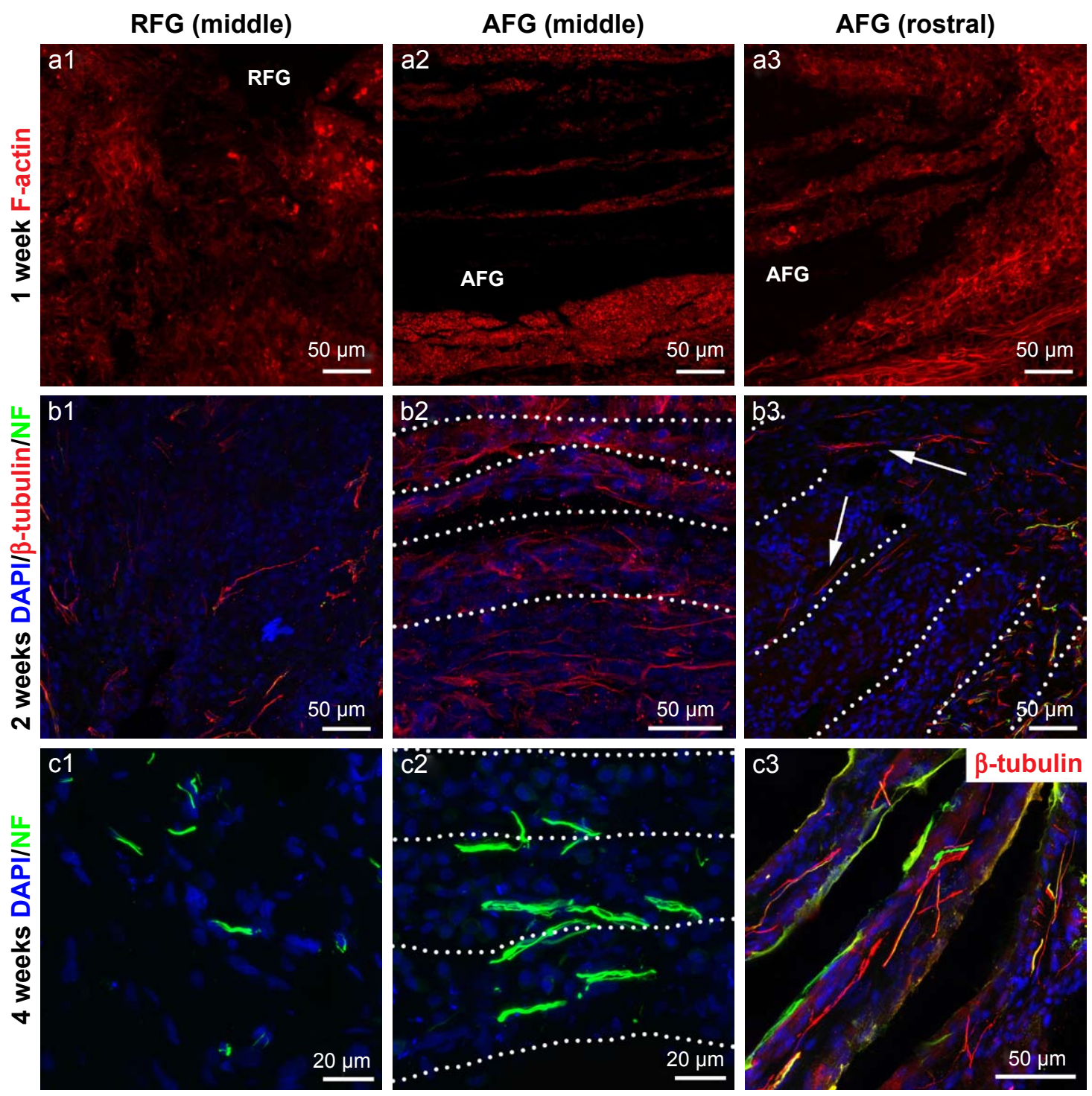

Figure 3 Aligned tissue cables formed by the endogenous cell invasion promote extensive axonal regeneration. (a I-a3) F-actin immunofluorescence staining at I week after surgery. The representative immunofluorescence staining images of $\beta$-tubulin III (red), NF (green), and DAPI (blue) at 2 weeks (bI-b3) and 4 weeks (cl-c3) after surgery. The dashed lines indicate the long axis of the aligned AFG and cell cables. The arrows in b3 show the cells (neurons) labeled by $\beta$-tubulin III migrate into the regrowth tissue. Abbreviations: AFG, aligned fibrin hydrogel; DAPI, 4',6-diamidino-2-phenylindole; NF, neurofilament; RFG, random fibrin hydrogel.

aligned and compact organization bridging the spinal cord defect, as shown in high-magnification images of the AFG group (Figure 4A[c2 and c3]). The quantification of NFpositive fibers in the injury site also proved the AFG scaffold could promote the axon aligned regrowth. As shown in Figure 4B, the average density of NF-positive fibers was higher in the injury site with the AFG scaffold implantation. Moreover, most of the fibers in the RFG group were less than $40 \mu \mathrm{m}$ (about 69\%), as shown in Figure 4C. The frequency distribution of fiber length in $\mathrm{AFG}$ group is shown in Figure 4D, and most of the fibers were longer, especially the percentage of long fibers $(>100 \mu \mathrm{m})$ was about $24 \%$, which was notably higher than the RFG group.

\section{Axon remyelination}

At 16 weeks, tissues in the middle of the damaged area were examined by TEM, as shown in Figure 5. In the control group, some of the axons appeared degenerated with degraded myelin sheath, as indicated by the arrow in Figure 5A(a1). Simultaneously, cells with abundant incompact ECM were observed in the injured region, which maybe was the glial scar (Figure 5A[a1 and a2]). In the RFG and AFG groups, the unmyelinated or myelinated axons as well as blood vessels could be clearly seen. The axon density and the thickness of myelin sheath were quantitatively calculated based on the TEM images (Figure 5B and C). The regrowth axon density in RFG and AFG groups had no obvious difference, but was significantly higher than that in control group. 
A
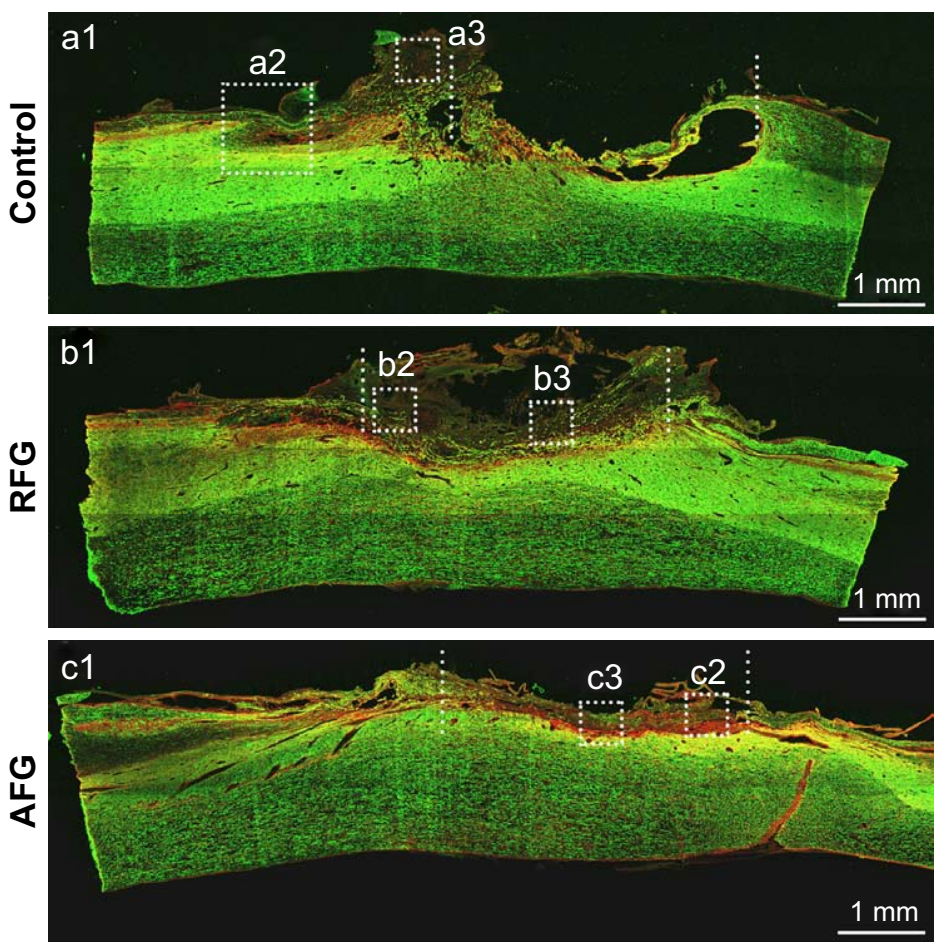

B

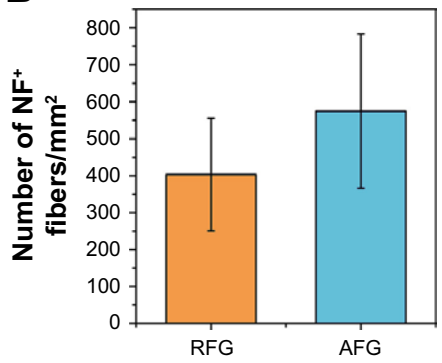

C

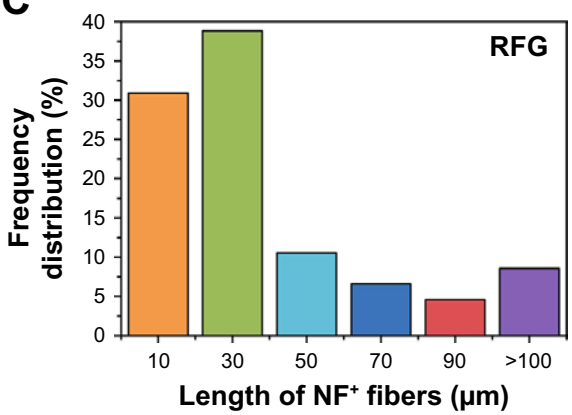

NF/GAP-43/DAPI
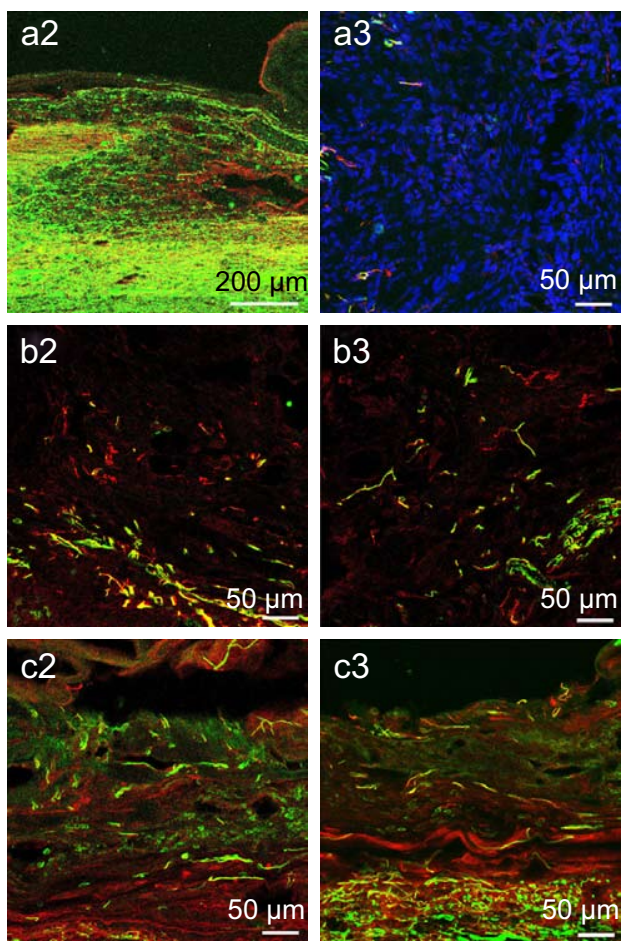

D

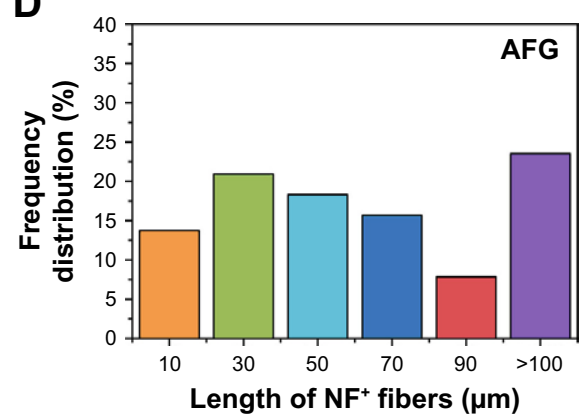

Figure 4 Nerve regeneration in the dorsal hemisected site at 8 weeks after surgery. Shown in Figure 5A, (al-cl) are the immunofluorescence staining images of T8-TI0 sagittal sections with NF (green), GAP43 (red), and DAPI (blue). Higher magnification images of the areas circled by the dotted white squares in the left images are shown in the right columns ([a2-a3], [b2-b3] and [c2-c3], respectively). The quantification of NF-positive fibers at the injury site is shown in (B), (C), and (D): (B) the density of NF-positive fibers at the injury site; the frequency distribution of NF-positive fibers of RFG (C) and AFG (D). Four individual rats per group were used for statistical analysis $(n=4)$, and five confocal images per rats were used for the NF-positive fiber counts and length calculation.

Abbreviations: AFG, aligned fibrin hydrogel; DAPI, 4',6-diamidino-2-phenylindole; NF, neurofilament; RFG, random fibrin hydrogel.

While the thickness of myelin sheath of the regrowth axons in the AFG group was much more than that of RFG group, which reflected better remyelination of the regrowth nerves in the AFG group.

\section{Nerve tissue regrowth identified by FA maps and DTI results}

The FA value is an anisotropic parameter: a numerical value between 0 (isotropic) and 1 (anisotropic), and values closer to 1 represent a more anisotropic structure, which appears as brighter areas of high anisotropy in FA maps. Normal spinal cord tissue appears bright in the FA maps because the nerve fibers have aligned structures. However, injured and irregular regrowth spinal cord tissues are dark in the FA maps because of their random structures. Thus, dark areas in the spinal cord FA maps can be used as a parameter for estimating lesion volume. ${ }^{42,43}$ The representative FA maps of the three groups are shown in Figure 6A, and the lesion sites implanted with different scaffolds are marked with red circles. The dark area in the control group map was the largest one among all the three groups, which suggested decreased anisotropy and a disconnected spinal cord at the lesion site. In comparison to the control and RFG groups, the FA maps of the AFGimplanted group appeared the brightest in the lesion site and 
A
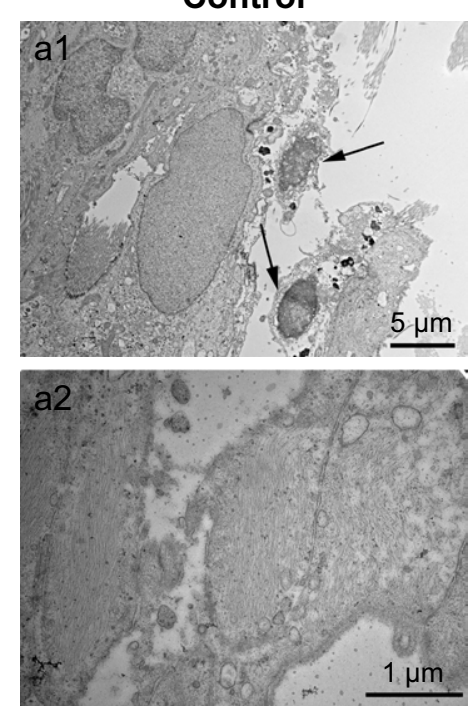

B

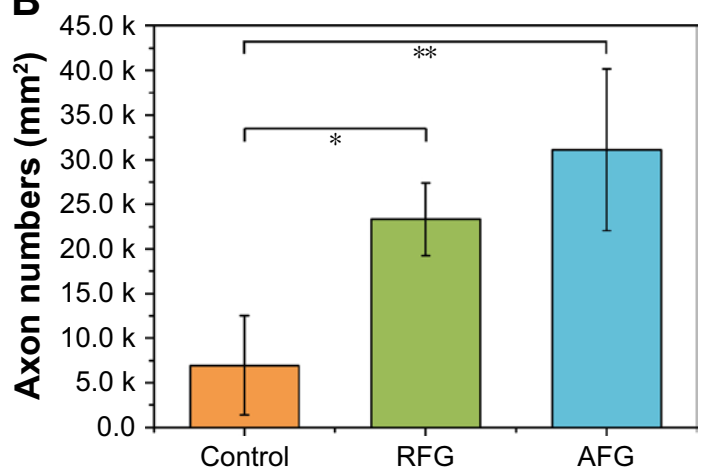

RFG
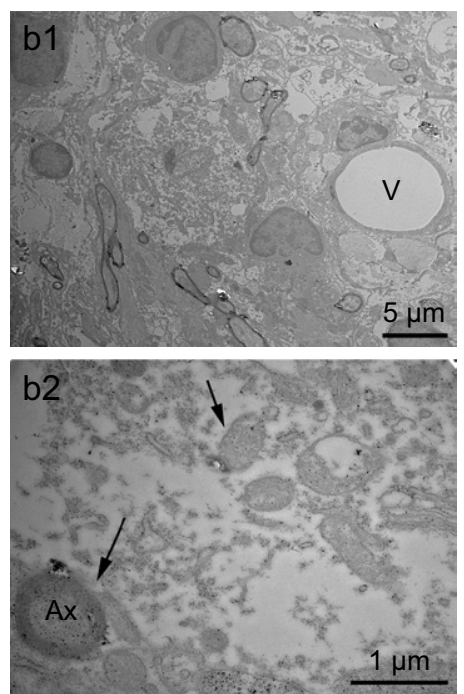

AFG
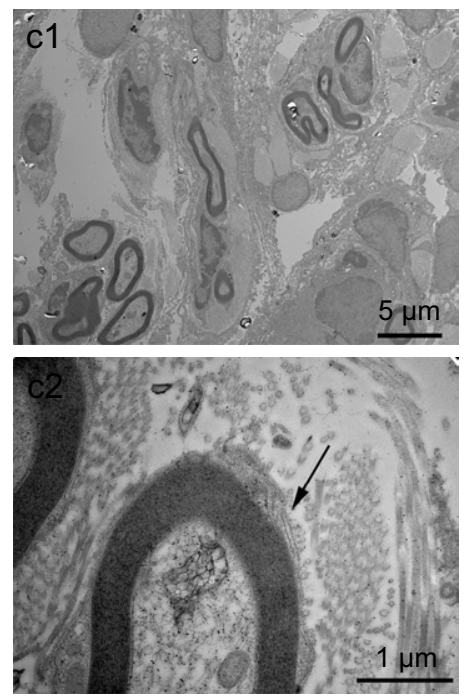

C

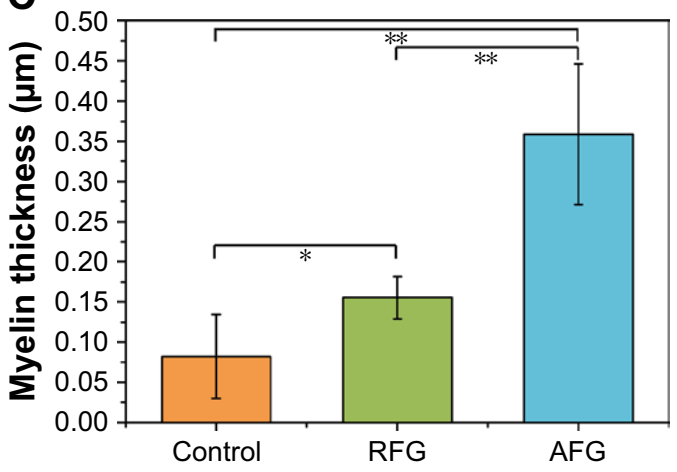

Figure 5 Transmission electron micrographs of the middle lesion site. (A) (al-a2) Axons with loose myelin sheaths and abundant collagen regenerated in the area of lesion site in the control group; compared to the control group, a great deal of myelinated axons with relatively compact myelin sheaths (Ax) are seen in the middle region of lesion site (cl-c2), and accompanying blood vessels (V) in the RFG group (bl-b2). The arrows show the axons. (B and $\mathbf{C}$ ) The number and myelin thickness of axons in the middle lesion site are summarized and analyzed. Data are expressed as mean \pm standard deviation. Statistical comparisons were performed using ANOVA, and $* p<0.05$, $* * p<0.0$ I. Four individual rats per group were used for statistical analysis $(n=4)$, and five TEM images per rat were used for the axon counts and myelin thickness calculation. Abbreviations: AFG, aligned fibrin hydrogel; ANOVA, analysis of variance; RFG, random fibrin hydrogel; TEM, transmission electron microscopy.

had the smallest dark area, indicating an improved diffusion tensor cylinder and enhanced aligned axonal regrowth.

DTI tractography was used as a qualitative indicator of SCI to visually track the aligned nerve fibers and thereby evaluate axonal regrowth in the lesion site. All of the DTI results were consistent with the FA maps. Figure 6A shows the DTI results of the left $(\mathrm{L})$ and posterior $(\mathrm{P})$ locations. The figures show no nerve fibers crossing the injury site from either the rostral or the caudal site in the control group as observed from the left side (Figure 6A[a4]). Furthermore, the nerve fibers did not even cross the area that was in the uninjured region of spinal cord, where the axons appeared degenerated and had lost their aligned fiber structure (Figure 6A[a5]). In the RFG group, although axon regrowth was observed from the histological examinations, the tracked nerve fibers were not across the lesion site yet (Figure 6B[b4]). However, the nerve fibers around the injury region could be tracked throughout the defect, indicating no degeneration occurred in the surrounding tissues (Figure 6B[b5]). Notably, in AFG group, abundant nerve fibers from the rostral and caudal locations could be tracked across the lesion site (Figure 6C[c4 and c5]).

Figure 6B shows the FA values as a function of distance. The area between the two dashed lines indicates the injured region. The FA values of the injured spinal cord in the control group were significantly lower than those in RFG and AFG groups in not only the injured region but also the neighboring areas due to the degeneration of axons. The FA values in the AFG group were higher than those of RFG group at the same position, although the difference was not that significant. These results indicate that the fibrin hydrogel implantation was beneficial for axon regrowth and decreased surrounding neural tissue degeneration. 

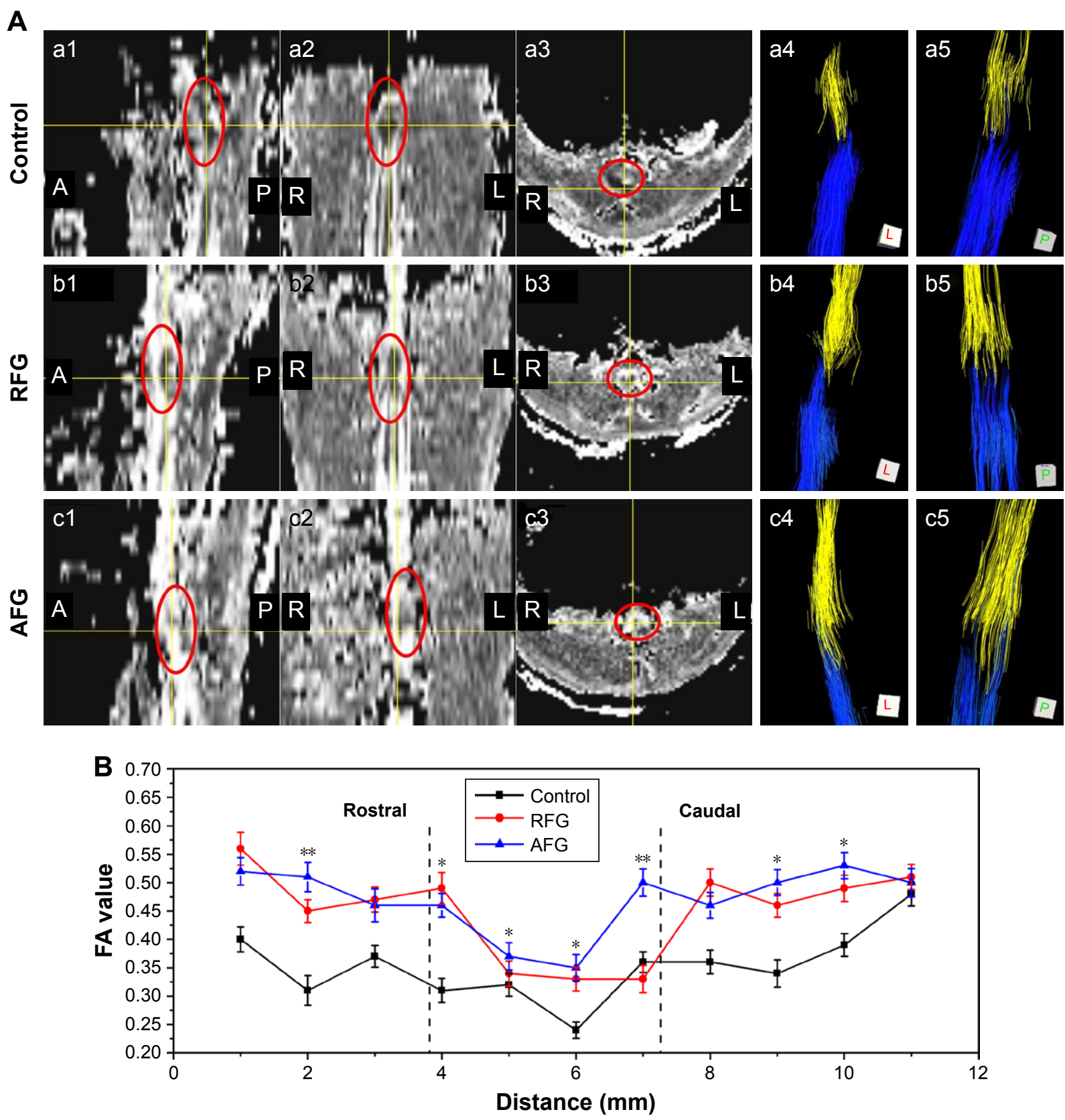

Figure 6 Representative FA maps, tractography images, and FA values of the spinal cord at 16 weeks after the implantation. (A) The bright region indicates high anisotropy in the representative FA maps ( $\mathrm{al}-\mathrm{a} 3$, bI-b3 and $\mathrm{cl}-\mathrm{c} 3$, where A, P, L, and R represent anterior, posterior, left, and right, respectively). In the images of (a4-a5), (b4-b5), and (c4-c5), yellow stands for fibers traced from the ROI rostral to the epicenter, where blue corresponds to fibers traced from the ROI caudal to the epicenter. (B) FA value versus location $(\mathrm{mm})$ reveals statistically significant differences between the AFG group and the RFG group at the lesion site $(n=4)$, ${ }^{*} p<0.05$, ${ }^{* *} p<0.01$. Furthermore, the FA value of AFG revealed statistically significant differences from control group animals at all locations along the spinal cord ( $p<0.0 \mathrm{I})$.

Abbreviations: AFG, aligned fibrin hydrogel; FA, fractional anisotropy; RFG, random fibrin hydrogel; ROI, region of interest.

\section{Recovery of locomotor function}

Locomotor function was evaluated using a serial BBB score analysis (Figure 7). At 1 week post-surgery, the average BBB scores were similar in all the experimental groups, indicating the loss of motor function after surgery. Then, the motor function in RFG and AFG groups recovered remarkably with rapidly increased BBB scores in comparison to the control group during the initial 4 weeks after the surgery. Moreover, the AFG group showed the best performance in locomotion among these three experimental groups with highest $\mathrm{BBB}$ scores until 8 weeks. The results indicated that the AFG implantation could promote the recovery of locomotor function at the early stage.

\section{Discussion}

The primary goal in spinal cord regeneration by using biomaterials is to promote axonal outgrowth and reduce barriers like the formation of cavities and glial scars. In order 


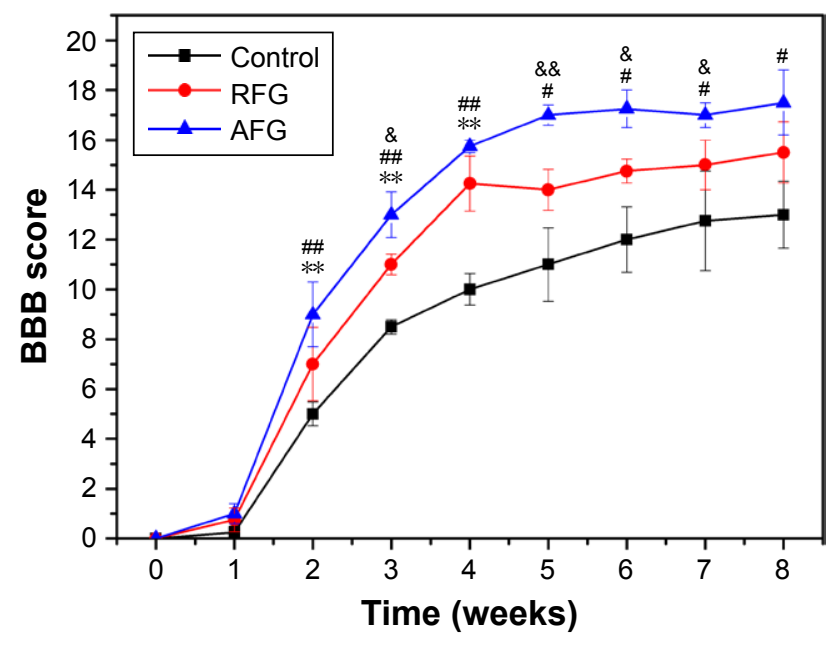

Figure 7 BBB score of the experimental groups. Data are expressed as mean \pm standard error of the mean ( $n \geq 8$ for each group). ${ }^{\#} p<0.05,{ }^{\# p}<0.01$ (AFG vs control at the same time point); ${ }^{* *} p<0.0$ I (RFG vs control at the same time point); ${ }^{\&} p<0.05$, ${ }^{2} p<0.0$ I (AFG vs RFG at the same time point).

Abbreviations: AFG, aligned fibrin hydrogel; BBB, Basso-Beattie-Bresnahan; RFG, random fibrin hydrogel.

to accomplish this goal, designing novel biomaterials that deliver biophysical and biochemical cues to modulate cellspecific responses and functions has been thought to be the key issue. In this study, the main chemical component of the hydrogels that we developed was fibrin, an important fibrous protein that has been widely proven to be beneficial for promoting axonal regrowth in both central and PNS regrowth. What is more, we here focused on two biophysical regulatory signals, low elasticity and structural alignment, both of which mimic the physical features of natural nerve tissues and show positive effects on enhancing axon regrowth in vivo.

A

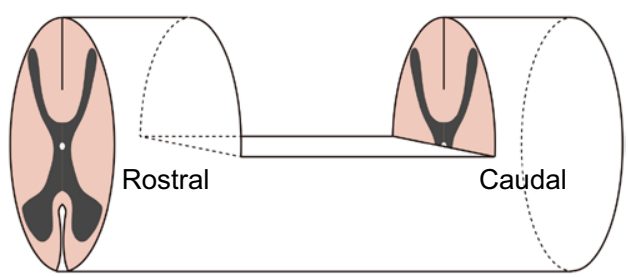

T9 dorsal hemisection spinal cord injury

C

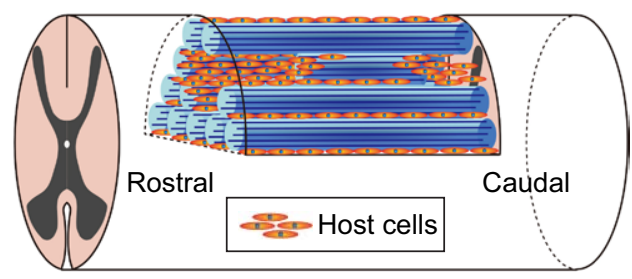

Host cells invasion and proliferation to replace the $A F G$
Thus, according to the biomimetic strategy, a hierarchically aligned fibrillar fibrin hydrogel with the characteristics of both aligned topography and low elasticity was designed and fabricated for the nerve regrowth in rat SCI.

Unlike CNS injury, spontaneous axonal regeneration and recovery can occur in the PNS. At the initial stage of PNS regeneration with the help of nerve guidance conduits, aligned fibrin cables form spontaneously to promote migration, longitudinal alignment, and proliferation of the various endogenous cells, forming a cable of biological tissue to guide axonal sprouting. ${ }^{39,44}$ Inspired by this finding, we therefore developed a hierarchically aligned fibrillar fibrin hydrogel through electrospinning technology and self-assembling process to mimic the component, microstructure, and functions of the native fibrin cables. In our previous study, the AFG has been proven to promote axonal outgrowth and prompt peripheral nerve regeneration in a rat sciatic nerve injury model. ${ }^{34,45}$ Therefore, here we applied the AFG for SCI repair by bridging the defect, which was estimated to promote the axon regrowth and restore motor functions. Our results showed that the fibrin hydrogels could rapidly induce endogenous cell invasion and proliferation into the defects with promoted axon regrowth and remyelination in comparison to the blank control group, which indicated the crucial importance of biomaterials. Besides, the invaded cells along the AFG fibers were organized in an oriented manner instead of a random distribution in RFG, thus forming aligned tissue cables to bridge the lesion site, which then guided axon regrowth and facilitated axon myelination. Figure 8 schematically illustrates the proposed mechanism for the

B

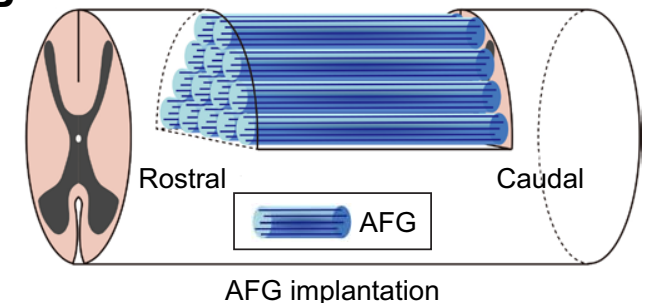

D

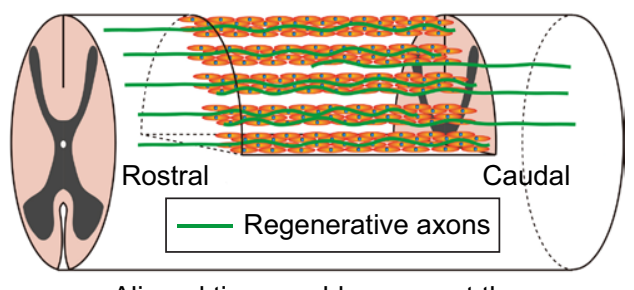

Aligned tissue cables support the axons' regeneration

Figure 8 Summarized schematic of regrowth process with AFG implantation in rat dorsal semi-section SCl model. For the T9 dorsal hemisection SCI (A), the AFG scaffold was implanted into the injury site to bridge the gap (B). Then, the host cells migrated into the AFG scaffold to replace the aligned structure (C) which supports the axons' regeneration (D).

Abbreviations: AFG, aligned fibrin hydrogel; SCI, spinal cord injury. 
axon regrowth with the involvement of the AFG scaffold. As one of the proteins involved in wound healing and PNS regeneration, the fibrin scaffold showed excellent integration with the host tissue, which was beneficial for endogenous cell invasion into the scaffold. At 2 weeks after implantation, the AFG scaffold was almost replaced by the invaded host cells, forming aligned tissue cables. Abundant blood vessels constructed concurrently within the scaffold to provide nutrition and oxygen for the regenerating tissues. After that, the "alive" aligned cables enable axon sprouting and support regrowth. Briefly, the AFG built a beneficial microenvironment in the lesion site that was greatly advantageous for further tissue regrowth and locomotor function recovery.

Following the primary injury of CNS, reactive astrocytes and an influx of inflammatory cells cause the expansion of the lesion cavity and proteoglycan upregulation to form the glial scar that presents a mechanical barrier to growing axons, leading to the secondary injury. Therefore, fast cell invasion into the lesion site to form a native parenchymal tissue instead of scar tissue largely determines the success of the following axonal regrowth and functional recovery. In comparison to RFG and control groups, the AFG could accelerate tissue invasion directionally in the early stage of repair. As the aligned tissue cables rapidly formed to replace the scaffold in the lesion site, the axons regrew with higher density and better myelination (confirmed by the histological examinations and DTI tracking), which contributed to the quicker motor functional recovery. Although the motor function of rats in the control groups partly recovered 8 weeks later, that should probably be attributed to the stronger regrowth capacity of rat CNS and the compensation of ventral nervous function. In our previously published work, the AFG also could promote axon regrowth and hindlimb motor function recovery in canine hemisected SCI model. ${ }^{46}$

\section{Conclusion}

An aligned fibrin scaffold with low elasticity and hierarchically oriented structure was fabricated and implanted into a T9 dorsal hemisected injury to promote axon regrowth. The results showed that the AFG scaffold induced the alignment, migration, and proliferation of host cells in the implanted hydrogel to replace the structure of the AFG scaffold. Aligned tissue cables then formed at the lesion site to bridge the injury stumps from the rostral to caudal sites. These aligned cables provided physical and chemical cues for aligned axonal growth and accelerating the recovery of locomotor function in the early stages. Furthermore, the myelination of the regrowth axons was obviously improved in the AFG group with larger density of regrowth axons and the thickness of myelin sheath. This work demonstrated the remarkable potential of the hierarchically organized AFG for axon regrowth and motor functional recovery following SCI.

\section{Acknowledgments}

This work was supported in part by the National Natural Science Foundation of China (number 31771056), China Postdoctoral Science Foundation (2016M591075), Fundamental Research Funds for the Central Universities (2302016FRF-TP-16-001A1), and Tsinghua University Initiative Scientific Research Program (20161080091).

\section{Disclosure}

The authors report no conflicts of interest in this work.

\section{References}

1. Khaing ZZ, Thomas RC, Geissler SA, Schmidt CE. Advanced biomaterials for repairing the nervous system: what can hydrogels do for the brain? Mater Today. 2014;17(7):332-340.

2. Papastefanaki F, Matsas R. From demyelination to remyelination: the road toward therapies for spinal cord injury. Glia. 2015;63(7): 1101-1125.

3. Mccreedy DA, Sakiyama-Elbert SE. Combination therapies in the CNS: engineering the environment. Neurosci Lett. 2012;519(2):115-121.

4. Yao S, Liu X, Wang X, Merolli A, Chen X, Cui F. Directing neural stem cell fate with biomaterial parameters for injured brain regeneration. Prog Nat Sci Mater. 2013;23(2):103-112.

5. Gumera C, Rauck B, Wang Y. Materials for central nervous system regeneration: bioactive cues. J Mater Chem. 2011;21(20):7033-7051.

6. Kim M, Park SR, Choi BH. Biomaterial scaffolds used for the regeneration of spinal cord injury (SCI). Histol Histopathol. 2014;29(11): 1395-1408.

7. He J, Wang XM, Spector M, Cui FZ. Scaffolds for central nervous system tissue engineering. Front Mater Sci. 2012;6(1):1-25.

8. Reilly GC, Engler AJ. Intrinsic extracellular matrix properties regulate stem cell differentiation. J Biomech. 2009;43(1):55-62

9. Saha K, Keung AJ, Irwin EF, et al. Substrate modulus directs neural stem cell behavior. Biophys J. 2008;95(9):4426-4438.

10. Engler AJ, Sen S, Sweeney HL, Discher DE. Matrix elasticity directs stem cell lineage specification. Cell. 2006;126(4):677-689.

11. Wilems TS, Sakiyama-Elbert SE. Sustained dual drug delivery of anti-inhibitory molecules for treatment of spinal cord injury. J Control Release. 2015;213:103-111.

12. Kushchayev SV, Giers MB, Hom Eng D, et al. Hyaluronic acid scaffold has a neuroprotective effect in hemisection spinal cord injury. J Neurosurg Spine. 2016;25(1):114-124.

13. Günther MI, Weidner N, Müller R, Blesch A. Cell-seeded alginate hydrogel scaffolds promote directed linear axonal regeneration in the injured rat spinal cord. Acta Biomater. 2015;27:140-150.

14. Zeng X, Qiu XC, Ma YH, et al. Integration of donor mesenchymal stem cell-derived neuron-like cells into host neural network after rat spinal cord transection. Biomaterials. 2015;53:184-201.

15. Yang Z, Zhang A, Duan H, et al. NT3-chitosan elicits robust endogenous neurogenesis to enable functional recovery after spinal cord injury. Proc Natl Acad Sci U S A. 2015;112(43):13354-13359.

16. Winter CC, Katiyar KS, Hernandez NS, et al. Transplantable living scaffolds comprised of micro-tissue engineered aligned astrocyte networks to facilitate central nervous system regeneration. Acta Biomater. 2016;38:44-58. 
17. Mothe AJ, Tam RY, Zahir T, Tator CH, Shoichet MS. Repair of the injured spinal cord by transplantation of neural stem cells in a hyaluronan-based hydrogel. Biomaterials. 2013;34(15):3775-3783.

18. Austin JW, Kang CE, Baumann MD, et al. The effects of intrathecal injection of a hyaluronan-based hydrogel on inflammation, scarring and neurobehavioural outcomes in a rat model of severe spinal cord injury associated with arachnoiditis. Biomaterials. 2012;33(18):4555-4564.

19. Estrada V, Brazda N, Schmitz C, et al. Long-lasting significant functional improvement in chronic severe spinal cord injury following scar resection and polyethylene glycol implantation. Neurobiol Dis. 2014;67: 165-179.

20. Silva NA, Sousa N, Reis RL, Salgado AJ. From basics to clinical: a comprehensive review on spinal cord injury. Prog Neurobiol. 2014; 114:25-57.

21. Pires LR, Pêgo AP. Bridging the lesion - engineering a permissive substrate for nerve regeneration. Regen Biomater. 2015;2(3):203-214.

22. Gao M, Lu P, Bednark B, et al. Templated agarose scaffolds for the support of motor axon regeneration into sites of complete spinal cord transection. Biomaterials. 2013;34(5):1529-1536

23. Thomas AM, Kubilius MB, Holland SJ, et al. Channel density and porosity of degradable bridging scaffolds on axon growth after spinal injury. Biomaterials. 2013;34(9):2213-2220.

24. Gros T, Sakamoto JS, Blesch A, Havton LA, Tuszynski MH. Regeneration of long-tract axons through sites of spinal cord injury using templated agarose scaffolds. Biomaterials. 2010;31(26):6719-6729.

25. Prang P, Müller R, Eljaouhari A, et al. The promotion of oriented axonal regrowth in the injured spinal cord by alginate-based anisotropic capillary hydrogels. Biomaterials. 2006;27(19):3560-3569.

26. Pawar K, Mueller R, Caioni M, et al. Increasing capillary diameter and the incorporation of gelatin enhance axon outgrowth in alginate-based anisotropic hydrogels. Acta Biomater. 2011;7(7):2826-2834.

27. Jiang X, Cao HQ, Shi LY, Ng SY, Stanton LW, Chew SY. Nanofiber topography and sustained biochemical signaling enhance human mesenchymal stem cell neural commitment. Acta Biomater. 2012;8(3): 1290-1302.

28. Lim SH, Liu XY, Song H, Yarema KJ, Mao HQ. The effect of nanofiberguided cell alignment on the preferential differentiation of neural stem cells. Biomaterials. 2010;31(34):9031-9039.

29. Han Q, Sun W, Lin H, et al. Linear ordered collagen scaffolds loaded with collagen-binding brain-derived neurotrophic factor improve the recovery of spinal cord injury in rats. Tissue Eng Part A. 2009;15(10): 2927-2935.

30. Han Q, Jin W, Xiao Z, et al. The promotion of neural regeneration in an extreme rat spinal cord injury model using a collagen scaffold containing a collagen binding neuroprotective protein and an EGFR neutralizing antibody. Biomaterials. 2010;31(35):9212-9220.

31. Han S, Wang B, Jin W, et al. The linear-ordered collagen scaffold-BDNF complex significantly promotes functional recovery after completely transected spinal cord injury in canine. Biomaterials. 2015;41:89-96.
32. Nguyen LH, Gao M, Lin J, Wu W, Wang J, Chew SY. Three-dimensional aligned nanofibers-hydrogel scaffold for controlled non-viral drug/gene delivery to direct axon regeneration in spinal cord injury treatment. Sci Rep. 2017;7:42212.

33. Berns EJ, Sur S, Pan L, et al. Aligned neurite outgrowth and directed cell migration in self-assembled monodomain gels. Biomaterials. 2014; 35(1):185-195.

34. Yao S, Liu X, Yu S, et al. Co-effects of matrix low elasticity and aligned topography on stem cell neurogenic differentiation and rapid neurite outgrowth. Nanoscale. 2016;8(19):10252-10265.

35. Pittier R, Sauthier F, Hubbell JA, Hall H. Neurite extension and in vitro myelination within three-dimensional modified fibrin matrices. J Neurobiol. 2005;63(1):1-14.

36. Khaing ZZ, Schmidt CE. Advances in natural biomaterials for nerve tissue repair. Neurosci Lett. 2012;519(2):103-114.

37. Ahmed TA, Dare EV, Hincke M. Fibrin: a versatile scaffold for tissue engineering applications. Tissue Eng Part B Rev. 2008;14(2):199-215.

38. Rajangam T, An SS. Fibrinogen and fibrin based micro and nano scaffolds incorporated with drugs, proteins, cells and genes for therapeutic biomedical applications. Int J Nanomedicine. 2013;8:3641-3662.

39. Daly W, Yao L, Zeugolis D, Windebank A, Pandit A. A biomaterials approach to peripheral nerve regeneration: bridging the peripheral nerve gap and enhancing functional recovery. $J R$ Soc Interface. 2012; 9(67):202-221.

40. Hyatt AJ, Wang D, Van Oterendorp C, Fawcett JW, Martin KR. Mesenchymal stromal cells integrate and form longitudinally-aligned layers when delivered to injured spinal cord via a novel fibrin scaffold. Neurosci Lett. 2014;569:12-17.

41. Zhang S, Liu X, Barreto-Ortiz SF, et al. Creating polymer hydrogel microfibres with internal alignment via electrical and mechanical stretching. Biomaterials. 2014;35(10):3243-3251.

42. Wu W, Zhao H, Xie B, et al. Implanted spike wave electric stimulation promotes survival of the bone marrow mesenchymal stem cells and functional recovery in the spinal cord injured rats. Neurosci Lett. 2011; 491(1):73-78.

43. Kelley BJ, Harel NY, Kim CY, et al. Diffusion tensor imaging as a predictor of locomotor function after experimental spinal cord injury and recovery. J Neurotrauma. 2014;31(15):1362-1373.

44. Belkas JS, Shoichet MS, Midha R. Peripheral nerve regeneration through guidance tubes. Neurol Res. 2004;26(2):151-160.

45. Du J, Liu J, Yao S, et al. Prompt peripheral nerve regeneration induced by a hierarchically aligned fibrin nanofiber hydrogel. Acta Biomater. 2017;55:296-309.

46. Zhang Z, Yao S, Xie S, et al. Effect of hierarchically aligned fibrin hydrogel in regeneration of spinal cord injury demonstrated by tractography: a pilot study. Sci Rep. 2017;7:40017.
International Journal of Nanomedicine

\section{Publish your work in this journal}

The International Journal of Nanomedicine is an international, peerreviewed journal focusing on the application of nanotechnology in diagnostics, therapeutics, and drug delivery systems throughout the biomedical field. This journal is indexed on PubMed Central, MedLine, CAS, SciSearch $®$, Current Contents $\AA /$ Clinical Medicine,

\section{Dovepress}

Journal Citation Reports/Science Edition, EMBase, Scopus and the Elsevier Bibliographic databases. The manuscript management system is completely online and includes a very quick and fair peer-review system, which is all easy to use. Visit http://www.dovepress.com/ testimonials.php to read real quotes from published authors. 